ARTICLE

Received 31 Oct 2012 | Accepted 15 Apr 2013 | Published 14 May 2013

DOI: $10.1038 /$ ncomms2868

\title{
A mutation in the receptor Methoprene-tolerant alters juvenile hormone response in insects and crustaceans
}

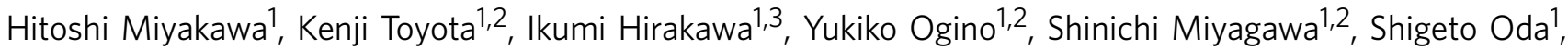
Norihisa Tatarazako ${ }^{4}$, Toru Miura ${ }^{5}$, John K. Colbourne ${ }^{6}$ \& Taisen Iguchi, ${ }^{1,2}$

Juvenile hormone is an essential regulator of major developmental and life history events in arthropods. Most of the insects use juvenile hormone III as the innate juvenile hormone ligand. By contrast, crustaceans use methyl farnesoate. Despite this difference that is tied to their deep evolutionary divergence, the process of this ligand transition is unknown. Here we show that a single amino-acid substitution in the receptor Methoprene-tolerant has an important role during evolution of the arthropod juvenile hormone pathway. Microcrustacea Daphnia pulex and D. magna share a juvenile hormone signal transduction pathway with insects, involving Methoprene-tolerant and steroid receptor coactivator proteins that form a heterodimer in response to various juvenoids. Juvenile hormone-binding pockets of the orthologous genes differ by only two amino acids, yet a single substitution within Daphnia Met enhances the receptor's responsiveness to juvenile hormone III. These results indicate that this mutation within an ancestral insect lineage contributed to the evolution of a juvenile hormone III receptor system.

\footnotetext{
${ }^{1}$ Okazaki Institute for Integrative Bioscience, National Institute for Basic Biology, National Institutes of Natural Sciences, Higashiyama, Myodaiji, Okazaki 4448787, Japan. ${ }^{2}$ Department of Basic Biology, School of Life Science, Graduate University for Advanced Studies (Sokendai), Nishigonaka, Myodaiji, Okazaki 444-8585, Japan. ${ }^{3}$ Ecogenomics, Inc., 1-1 Hyakunenkouen, Kurume, Fukuoka 839-0864, Japan. ${ }^{4}$ Environmental Quality Measurement Section, Research Center for Environmental Risk, National Institute for Environmental Studies, 16-2 Onogawa, Tsukuba, Ibaraki 305-8506, Japan. ${ }^{5}$ Laboratory of Ecological Genetics, Graduate School of Environmental Science, Hokkaido University, Sapporo 060-0810, Japan. ${ }^{6}$ School of Biosciences, University of Birmingham, Edgbaston, Birmingham B15 2TT, UK. Correspondence and requests for materials should be addressed to T.I. (email: taisen@nibb.ac.jp).
} 
uvenile hormones (JHs) are acyclic sesquiterpenoid hormones that regulate important physiological and developmental processes among arthropods, including metamorphosis, moulting, growth, reproduction, sex and caste determination $^{1-7}$. As exposure of JHs to animals can easily deflect developmental pathways, JHs are well studied in crustaceans for aquaculture $^{8-10}$ and in insects for agricultural pest control ${ }^{11,12}$. Crustaceans mainly use methyl farnesoate (MF) as the innate $\mathrm{JH}$ ligand ${ }^{13}$ whereas insects use an epoxide form of MF called JH III (JH III), which is catalysed by the MF epoxidase, CYP15A1 (ref. 14). Therefore, MF is the precursor form of active $\mathrm{JH}$ in insects ${ }^{15-17}$. However, little is known about their receptor systems and how they evolved.

Freshwater microcrustaceans Daphnia pulex and D. magna show striking JH-mediated polyphenisms such as environmental sex determination, induction of haemoglobin in response to reduced oxygen availability in aquatic habitats and inducible morphological defenses against predators signalled by kairomones ${ }^{8-10,18-21}$. In particular, the relationship between environmental sex determination and $\mathrm{JH}$ has been well studied over the last decade ${ }^{8-10,22}$. Most Daphnia species usually produce female offspring by parthenogenesis; however, once environmental conditions become barren, such as starvation or crowding, they produce male offspring and sexual reproduction occurs ${ }^{23}$. Male production is also induced by topical application of $\mathrm{JH}$ and $\mathrm{JH}$ analogues independent of environmental condition ${ }^{8-10}$, suggesting that $\mathrm{JH}$ is a key endocrine factor for sex determination working downstream of environmental stimuli in Daphnia. To date, several chemicals, including MF, JH III and four artificially synthesized juvenoids used in this study (that is, fenoxycarb, pyriproxyfen, methoprene and epofenonane), have been shown to be able to induce male daphniids with various concentration ranges ${ }^{10,22}$ (Table 1 ). Although receptors of $\mathrm{JH}$ are still unknown in any of the crustacean species, basic information obtained from these ecotoxicological studies is helpful in understanding crustacean $\mathrm{JH}$ pathway. There is every possibility that the difference in the capacity to induce male, shown by these chemicals, reflects ligand selectivity of $\mathrm{JH}$ receptor systems of Daphnia directly.

Methoprene-tolerant (Met) and steroid receptor coactivator (SRC) are proteins that belong to the basic helix-loop-helix (bHLH)-Per-Arnt-Sim (PAS) family of transcription factors. Both have important roles in the $\mathrm{JH}$ reception and downstream transcriptional activation ${ }^{24-26}$. Met is regarded as a leading candidate for the $\mathrm{JH}$ receptor in insect studies ${ }^{27-29}$. SRC-whose name is from the mammalian homologue SRC-1 with orthologs named FISC and Taiman in Aedes and Drosophila species-is also involved in the reception and signal transduction of $\mathrm{JH}^{24-26}$. Although Met exists as a homodimer (or heterodimer with the
Drosophila-specific paralog, germ cell-expressed (GCE)) in the absence of $\mathrm{JH}^{30}$, the homodimer is remodelled to form a heterodimer with SRC followed by transcriptional activation of downstream elements upon $\mathrm{JH}$ binding to the carboxy-terminal PAS domain (PAS-B) of $\mathrm{Met}^{24-26 .}$

To understand the molecular underpinnings of the $\mathrm{JH}$ reception system in daphniids and ligand transition that likely occurred when hexapods evolved from their pancrustacean ancestor $^{31}$, we tested the JH sensitivity of Met and SRC in two crustacean species, D. pulex and D. magna. As a result, Daphnia Met and SRC showed capability to respond to various juvenoid in different dose ranges, respectively. Moreover, we demonstrate that a single amino-acid substitution within Daphnia Met enhances the receptor's responsiveness to JH III, suggesting that this mutation contributed to the evolution of a JH III receptor system.

\section{Results}

Cloning and characterization of Daphnia Met and SRC. We cloned and characterized the full-length sequences of $\mathrm{Met}$ and $S R C$ genes from two Daphnia species, designated as Dappu-Met and Dappu-SRC for D. pulex, and Dapma-Met and Dapma-SRC for D. magna (accession numbers: AB698067-AB698070). Pairwise sequence identities of bHLH, PAS-A and PAS-B domains between Daphnia and selected insect species range from $15 \%$ to $78 \%$ (Fig. 1a-c). Six of eight polymorphic amino-acid residues within PAS-B domain forming JH-binding pockets are conserved in Met of both Daphnia species ${ }^{25}$ (Fig. 1d). At the other two sites, valine in insects is substituted for threonine in Daphnia, and threonine in insects is substituted by serine (Fig. 1d). We hypothesize that one or both amino-acid substitutions cause differences in ligand specificity of Met between crustaceans and insects. Both genes are required for development; transcriptional knockdown of Dapma-Met or Dapma-SRC result in embryonic death (Fig. 2 and Table 2).

JH-dependent heterodimerization of Daphnia Met and SRC. We tested juvenoid-dependent Met-SRC protein interactions in Daphnia by two-hybrid luciferase assay ${ }^{25,32}$ (see Methods). MF ( $\mathrm{JH}$ in crustaceans), $\mathrm{JH}$ III (JH in insects) and four artificially synthesized juvenoids (fenoxycarb, pyriproxyfen, methoprene and epofenonane) were used for this study. As described above, all these chemicals induce male offspring development in Daphnia ${ }^{10,22}$ (Table 1). We detected concentration-dependent luciferase activation from the heterodimerization of Met with SRC by all juvenoid treatments, except for epofenonane (Fig. 3a,b). Although the effective dose responses to the juvenoids vary for both species, parallel responses are observed

Table 1 | EC 50 of transcriptional activation via heterodimerization between SRC and Met (mutant) used in this study.

\begin{tabular}{|c|c|c|c|c|c|c|c|c|c|}
\hline & \multicolumn{4}{|c|}{ D. pulex } & \multicolumn{4}{|c|}{ D. magna } & \multirow[b]{2}{*}{$\begin{array}{l}\text { D. magna male } \\
\text { induction }\end{array}$} \\
\hline & wt & T292V & S323T & T292V, S323T & wt & T296V & S327T & T296V, S327T & \\
\hline Methyl farnesoate & $4.906 \mathrm{E}-07$ & $4.349 E-08$ & $3.631 E-07$ & $3.308 \mathrm{E}-08$ & $4.764 \mathrm{E}-07$ & $2.670 E-08$ & $2.064 \mathrm{E}-07$ & $2.372 \mathrm{E}-08$ & $2.777 \mathrm{E}-07$ \\
\hline $\mathrm{JH}$ III & $8.519 E-06$ & $6.056 \mathrm{E}-07$ & $6.096 \mathrm{E}-06$ & $7.681 \mathrm{E}-07$ & $5.707 E-06$ & $3.869 E-07$ & $5.207 E-06$ & $4.900 E-07$ & $4.009 E-06$ \\
\hline Fenoxycarb & $1.412 \mathrm{E}-08$ & & & & $1.068 \mathrm{E}-08$ & & & & $3.053 \mathrm{E}-09$ \\
\hline Pyriproxyfen & $8.880 E-07$ & & & & 5.183E107 & & & & $4.387 E-10$ \\
\hline Methoprene & $\mathrm{NA}^{\dagger}$ & $1.021 E-05$ & $\mathrm{NA}^{\dagger}$ & $4.937 E-06$ & $\mathrm{NA}^{\dagger}$ & $5.889 E-06$ & $\mathrm{NA}^{\dagger}$ & $4.296 E-06$ & $\mathrm{NA}^{\dagger}$ \\
\hline Epofenonane & & & & & $\mathrm{NA}^{\dagger}$ & & & & $2.108 E-07$ \\
\hline
\end{tabular}


a

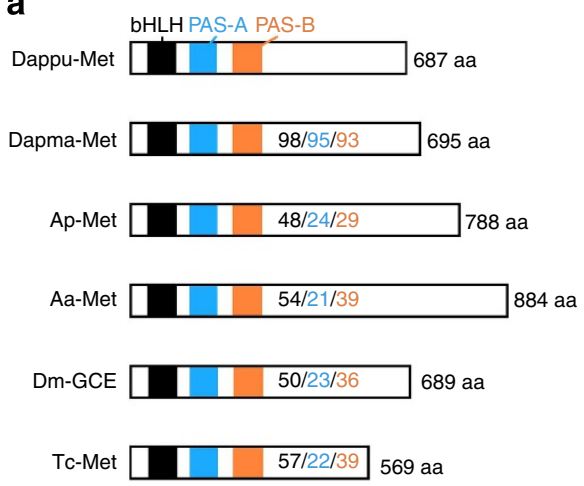

b

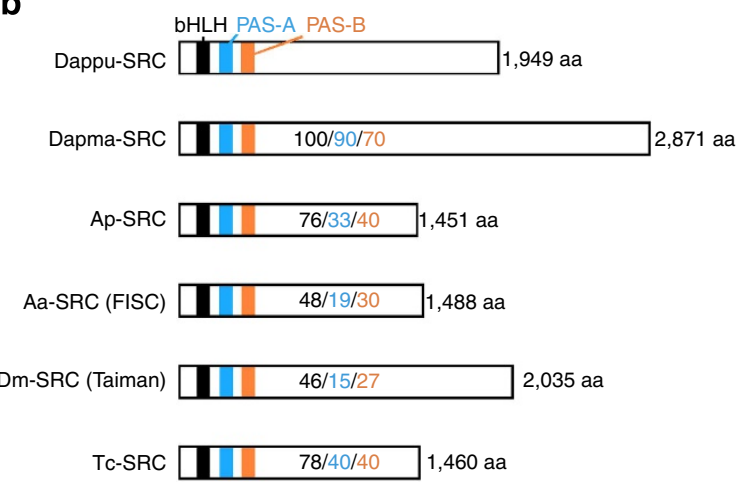

C

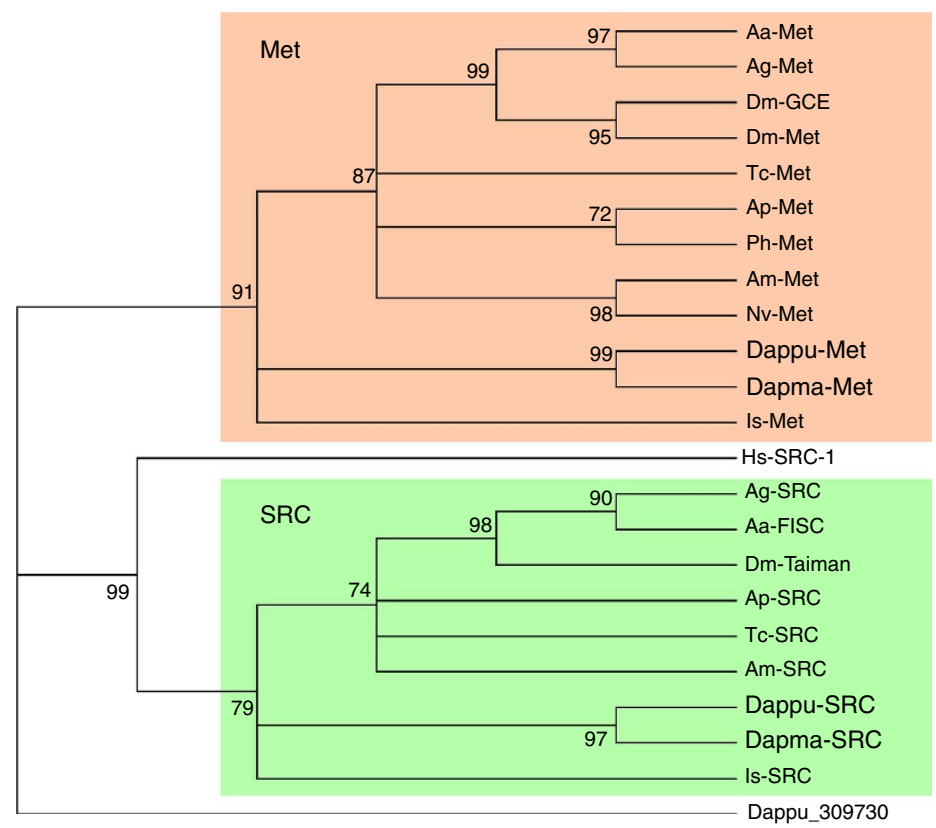

d

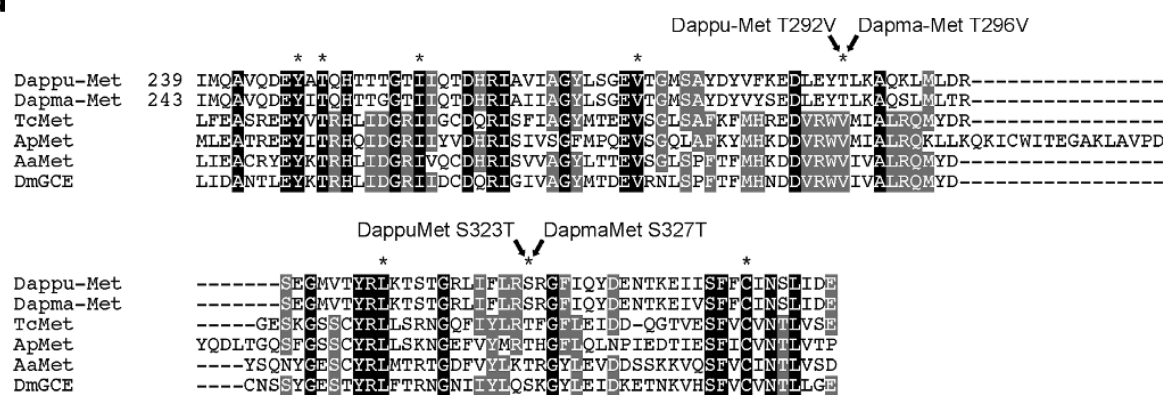

Figure 1 | Met and SRC in two Daphnia species. (a,b) Met and SRC in two Daphnia species, D. pulex (Dappu) and D. magna (Dapma), and homologues in insect species. Both Daphnia Met (a) and SRC (b) contain bHLH domain and PAS (PAS-A and PAS-B) domain in their sequences. Values in each sequence indicate percentage identity of amino-acid sequences of bHLH (black), PAS-A (blue) and PAS-B (orange) domain with those of $D$. pulex. Characteristic SRC family motifs LXXLL and glutamine-rich (Q-rich) regions were identified within the central and C-terminal regions of both Dappu-SRC and Dapma-SRC 39 . (c) Maximum Likelihood tree based on the amino-acid sequences of Met and SRC suggested that Daphnia Met and SRC were orthologs of insect Met and SRC, respectively. Dappu_309730 is another protein with bHLH-PAS domain found in the genome database of D. pulex. Strongly supported nodes ( $>70 \%$ ) are labelled with the percentage of bootstrap 1,000 replications. (d) Multiple alignment of PAS-B domain in Daphnia and insect Met. Asterisks indicate eight amino acids involved with $\mathrm{JH}$ III binding in Tribolium Met $^{24}$. In this study, two amino acids indicated by arrows were mutated to the corresponding amino acids used in insects. Species abbreviations and accession codes are as follows: Aedes aegypti (Aa) Met (XP_001660262.1) and SRC (FISC) (ABE99837.2); Anopheles gambiae (Ag) Met (XP_316059.4) and SRC (XP_001689140.1); Apis mellifera (Am) Met (XP_395005.4) and SRC (XP_394114.4); Acyrthocyphon pisum (Ap) Met (XP_003246905.1) and SRC (XP_001944363.2); D. magna (Dapma) Met (AB698069) and SRC (AB698070); D. pulex (Dappu) Met (AB698067) and SRC (AB698068); D. melanogaster (Dm) Met (NP_511126.2), GCE (NP_511160.2) and SRC (Taiman) (AAG16637.1); Homo sapiens (Hs) SRC-1 (AAB50242.1); Ixodes scapularis (Is) Met (XP_002410323.1) and SRC (XP_002402360.1); Nasonia vitripennis (Nv) Met (XP_001606775.2); Pediculus humanus (Ph) Met (XP_002430841.1); Tribolium castaneum (Tc) Met (EFA00995.1) and SRC (EFA04027.1). aa, amino acid. 


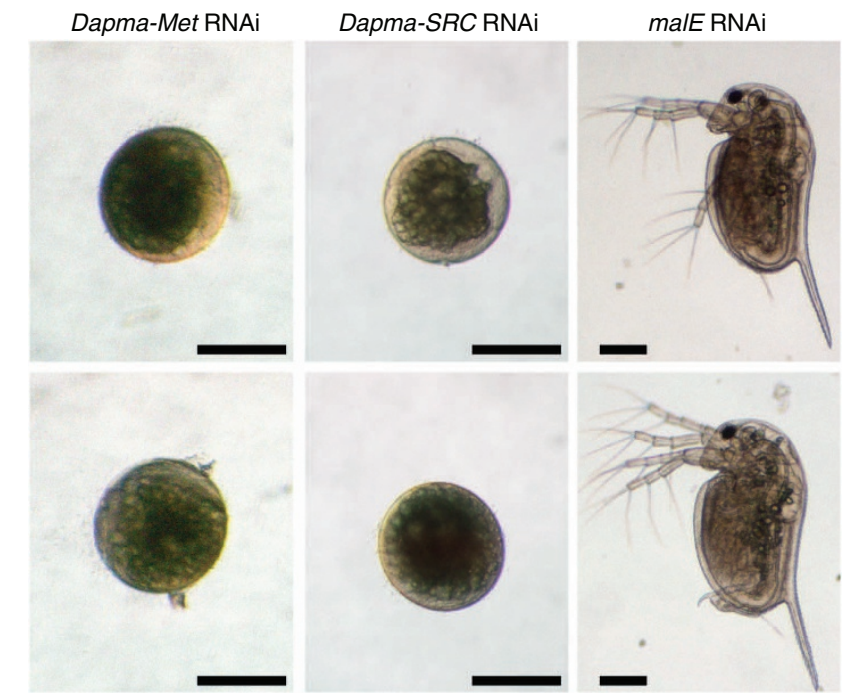

Figure 2 | Phenotypes of RNA interference (RNAi) targeting of the Dapma-Met and Dapma-SRC. Development of Dapma-Met or Dapma-SRC dsRNA-injected embryos was aborted at 20-30 h after oviposition. E. coli malE dsRNA-injected embryos grew into the first instar juveniles at $80 \mathrm{~h}$ after oviposition. Scale bars, $200 \mu \mathrm{m}$.

\section{Table 2 | Summary of the result of RNAi transcriptional} knockdown experiments.

Dapma-Met Dapma-SRC malE

\begin{tabular}{lccc}
\hline Number of aborted eggs/injected eggs & $11 / 13$ & $11 / 11$ & $0 / 6$ \\
Percentage of phenotypes & 84.6 & 100 & 0 \\
\hline
\end{tabular}

RNAi, RNA interference.

suggesting that the Met-SRC complex functions as a JH receptor in Daphnia species, as in insects ${ }^{24-26}$. In addition, the effect of $\mathrm{MF}$ on heterodimerization is nearly tenfold greater than that of $\mathrm{JH}$ III, which is consistent with the knowledge that crustaceans use MF as an active form of JH (Fig. 3a,b and Table 1), not JH III. Dappu-Met and Dapma-Met were also swapped in combination with Dapma-SRC and Dappu-SRC for each of the treatments. These swapped pairs also exhibited juvenoid-dependent dimerization (Fig. 5), yet combinations of both Met-Met and SRC-SRC did not show juvenoid-dependent responses (Fig. 5), thus further confirming that the Met and SRC protein complex functions as a $\mathrm{JH}$ receptor in daphniids.

Transition of $\mathrm{JH}$ ligand sensitivity. Finally, we tested our hypothesis that the threonine $\rightarrow$ valine and/or serine $\rightarrow$ threonine amino-acid substitutions in Met cause different ligand specificities of the receptor between crustaceans and insects, by measuring ligand-dependent heterodimerization using mutant Daphnia Met (Fig. 1d) that have amino acids T292V in DappuMet and T296V in Dapma-Met exchanged with Val-297 of Tribolium Met, and have amino acids S323T in Dappu-Met and S327T in Dapma-Met exchanged with Thr-330, also of the Tribolium gene (Fig. 1d). MF, JH III and methoprene treatments were used in this last experiment. For both Daphnia Met genes, the Val-297 mutation significantly increases the receptor sensitivity to JH III (Fig. 3c and Table 1). By contrast, mutations
S323T and S327T did not alter ligand sensitivity (Fig. 3c and Table 1).

\section{Discussion}

Although there are a lot of reports about the effect of juvenoids on environmental sex determination in daphniids ${ }^{8-10,22}$, their $\mathrm{JH}$ receptor molecules have been unclear to date. Here, we cloned Met and SRC from two Daphnia species, and demonstrated that Met and SRC proteins form heterodimer only in the presence of juvenoids, suggesting that these molecules act as a $\mathrm{JH}$ receptor in crustaceans as in insects ${ }^{24-26}$. Moreover, ligand selectivity that was estimated from the half maximal effective concentration $\left(\mathrm{EC}_{50}\right)$ values for heterodimerization was generally in accord with previous data for inducing male offspring development when testing six juvenoids $\left(\mathrm{EC}_{50}\right.$ values: fenoxycarb $<\mathrm{MF}<\mathrm{JH}$ III $<$ methoprene; Table 1 and Fig. 4) ${ }^{10,22}$. Although $\mathrm{EC}_{50}$ values of pyriproxyfen and epofenonane were not consistent between these two different experiments, the observed difference between the effective ranges for male offspring induction in the two species is likely caused by ligand selectivity of Met (or the Met-SRC complex) for juvenoids. Epofenonane is the only tested compound that does not induce heterodimerization, despite its effect at inducing male offspring development (Fig. 3a,b and Table 1). For most juvenoids, male induction is also associated with a great reduction of the number of offspring. In contrast, epofenonane can also induce male production with only a slight change in brood size $\mathrm{e}^{10,22}$. It is therefore likely that epofenonane induces male production by a different mechanism from the other juvenoids and their synthetic derivatives, which might not involve Met and SRC. On the other hand, in several insects, juvenoids lead to dissociation of Met-Met homodimer ${ }^{25,30}$, yet combinations of two Met plasmids in daphniids indicated neither homodimerization nor juvenoid-dependent responses (Fig. 5), thus further confirming that the Met and SRC protein complex functions as a $\mathrm{JH}$ receptor in daphniids.

For both Daphnia Met genes, the Val-297 mutation significantly increases the receptor sensitivity to JH III (Fig. $3 \mathrm{c}$ and Table 1), suggesting that amino-acid substitution to valine at this site had contributed to the transition of $\mathrm{JH}$ ligand during insect evolution. Furthermore, this amino-acid substitution to valine is thought to be involved in reception of methoprene, to which daphniids show lower sensitivity than insects, consistent with a previous report demonstrating that a single amino-acid mutation at Val-297 in Tribolium Met inhibited binding of methoprene to $\mathrm{Met}^{24}$. This mutation, however, also increases sensitivity to MF (Fig. $3 c$ and Table 1). Several studies report detectable amounts of MF in insect haemolymph, together with other juvenoids $7,33,34$. Further studies are required to determine whether both JH III and MF target the same insect receptor. By contrast, mutations S323T and S327T did not alter ligand sensitivity (Fig. 3c and Table 1), possibly because both serine and threonine have similar biochemical characteristics ${ }^{35}$. Given that a similar substitution to threonine can also be found within the Drosophila melanogaster GCE (Fig. 1d), it is thought to be a less functional polymorphism during the evolution of $\mathrm{JH}$ receptor.

In summary, present study strongly suggests that the Met-SRC complex operates as a $\mathrm{JH}$ receptor in daphniids as well as insects, but their ligand sensitivities are somewhat different from each other. It can be a reason why each juvenoid shows so varied effects and toxicity depending on animal species and taxa. Moreover, a single amino-acid substitution is revealed important during evolution of the arthropod $\mathrm{JH}$ pathway, by comparative functional genetic studies using a branchiopod crustacean model species. This knowledge, coupled with the identification of a JHsynthesizing organ and isolation of $\mathrm{JH}$-responsive elements 
a

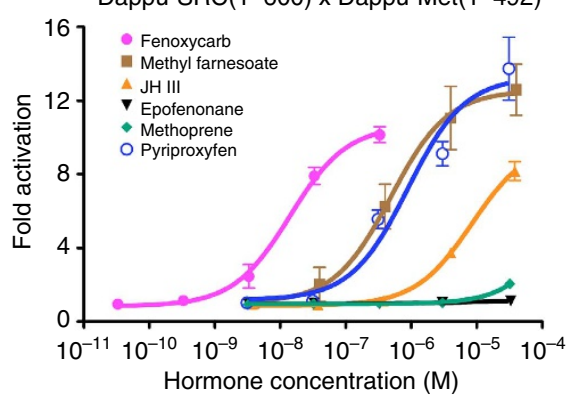

C
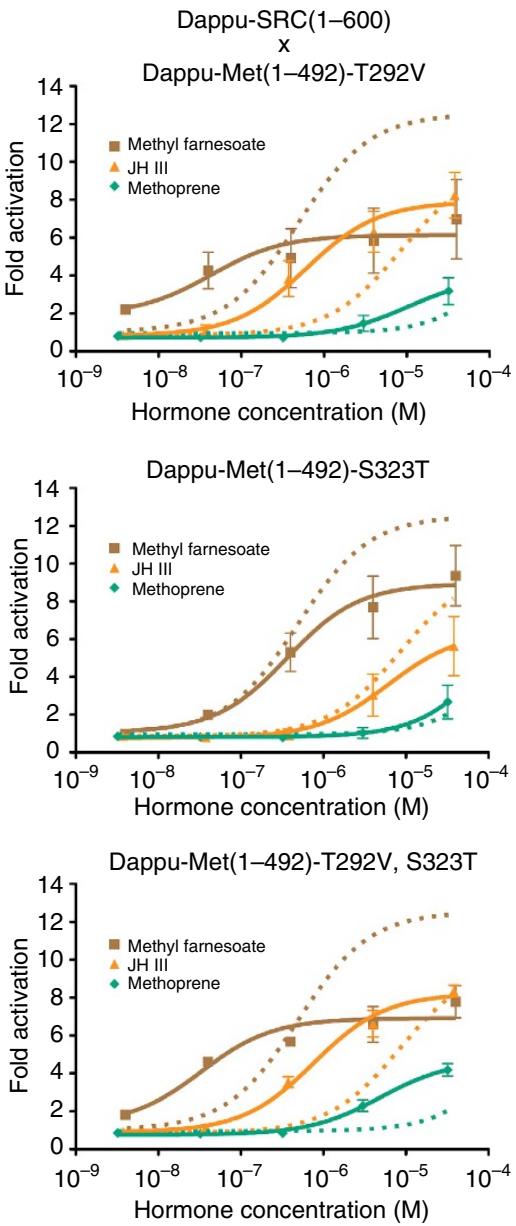

b

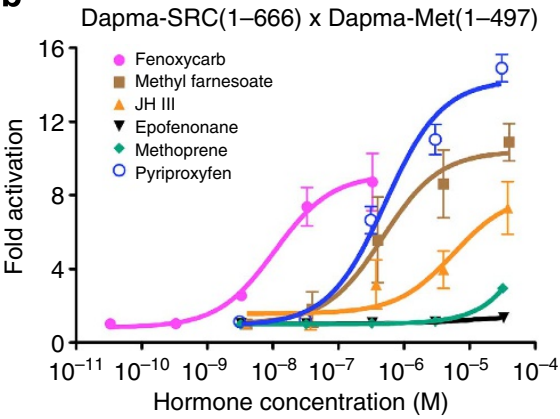

Hormone concentration $(\mathrm{M})$
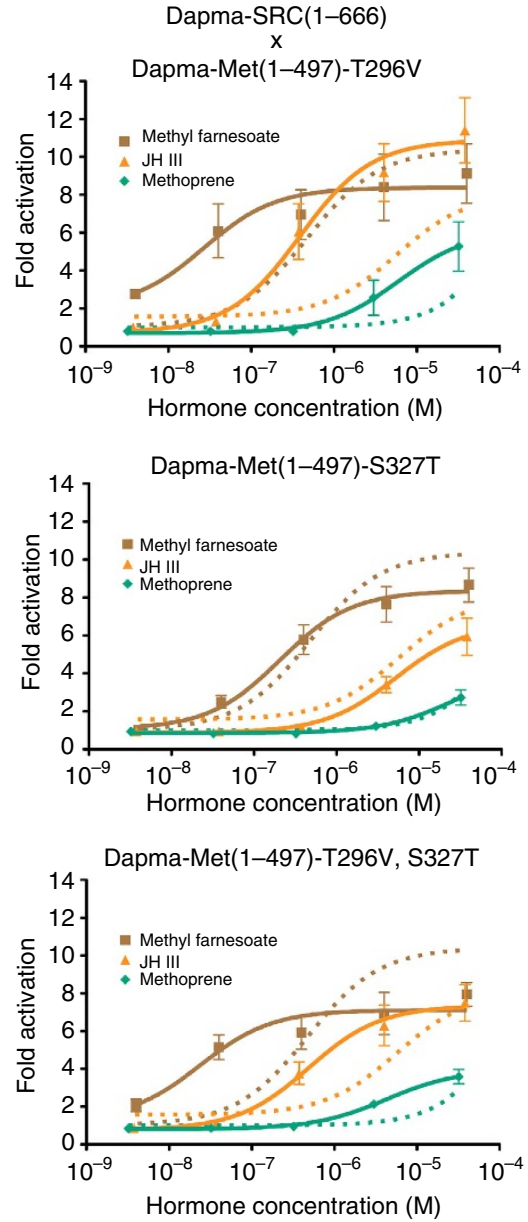

Figure 3 | Transcriptional activation via heterodimerization between Daphnia Met and SRC exposed to various juvenoids. (a) Concentration-response profile for SRC(1-600) and wild-type Met(1-492) of D. pulex. (b) Concentration-response profile for SRC(1-666) and wild-type Met(1-497) of D. magna. (c) Concentration-response profile for SRC and mutant Met. Vertical axis indicates fold activation, which is the luciferase activity normalized by solvent control $(0.1 \%$ ethanol) of each experiment. Dotted lines indicate profiles of wild types. Each point represents the mean of triplicate determinations, and vertical bars represent the mean \pm s.d.

interacting with Met-SRC complex, is expected to provide a more unified mechanistic understanding of the development and environmentally induced phenotypic plasticity across Arthropoda.

\section{Methods}

Animals. The D. pulex and D. magna clones used in the experiments were provided by the National Institute for Environmental Studies (NIES), Tsukuba, Japan. The clones were reared in the laboratory at $20^{\circ} \mathrm{C}$ in aged tap water and fed unicellular green algae (Chlorella Industry Co. Ltd, Fukuoka, Japan) over generations in 11 beakers in a temperature- and photocycle-controlled incubator $\left(20^{\circ} \mathrm{C}\right.$, 16-h light/8-h dark).

Cloning of Met and SRC. Amino-acid sequences of insect Met and SRC homologues were obtained using euGene's Arthropod genomes (http://insects.eugenes.org/arthropods/), and aligned by the ClustalX program ${ }^{36}$ using default options. Based on the aligned sequences, conserved regions were identified and used to perform TBLASTN searches against the wFleaBase (http://wfleabase.org/) to identify $D$. pulex gene models. PCR primers for cloning were designed based on 


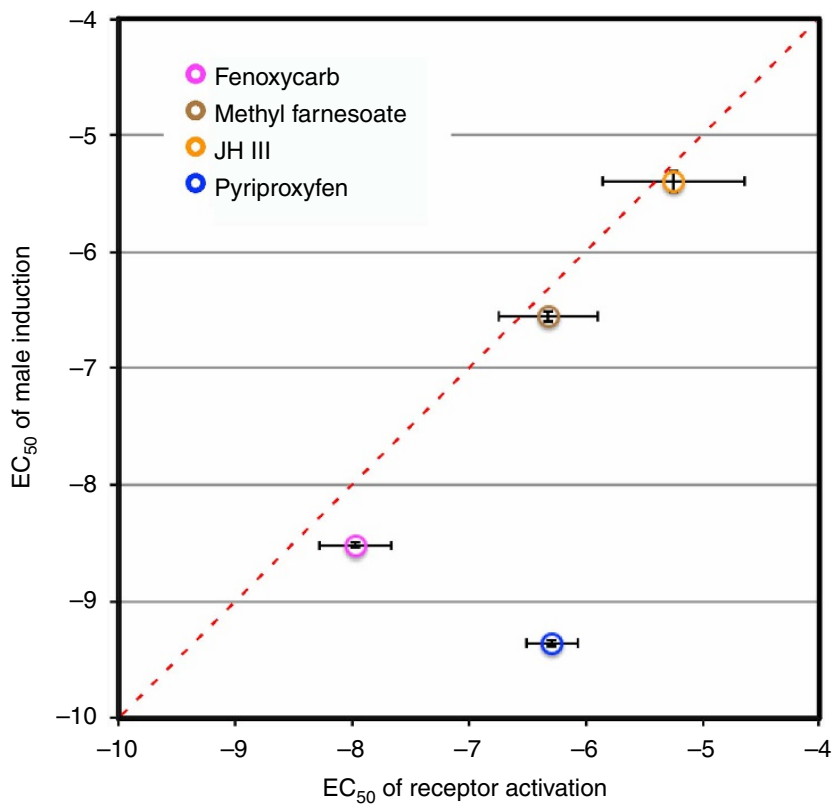

Figure 4 | Logarithmic relationships between $\mathrm{EC}_{50}$ of receptor activation and juvenoid induction of males in D. magna. Error bars indicate $95 \%$ confidence intervals. Dotted line indicates log ratio (1). $E C_{50}$ values of male induction are based on the data of previous studies (Tatarazako et al. ${ }^{10}$ and Oda et al. ${ }^{22}$ (see Table 1)).

sequences of these gene models. Total RNA was extracted from $\sim 20$ adult $D$. pulex and D. magna using an SV Total RNA Isolation System (Promega, Madison, WI) and converted to complementary DNA using Superscript III and random primers (Life Technologies, Carlsbad, CA) according to the manufacturer's protocol. Partial sequences of Met and SRC were obtained by PCR amplification, and full-length cDNAs were obtained by GeneRacer Kit (Life Technologies). Domain and motif searches were performed with obtained sequences using the European Bioinformatics Institute (EBI) InterPro database (http://www.ebi.ac.uk/Tools/ InterProScan/).

Phylogenetic analysis. The amino-acid sequences were aligned by the MEGA5 software using default options ${ }^{37}$. The Maximum Likelihood tree was constructed from this alignment using a 1,000 replicate bootstrap analysis, along with complete deletion options.

RNA interference. Knockdown of Dapma-Met and Dapma-SRC was performed using RNA interference technique according to our previous study ${ }^{38}$. Escherichia coli malE gene was used as a negative control. Double-stranded RNA (dsRNA) was synthesized using the MEGAscript RNAi Kit (Life Technologies). Eggs were obtained from D. magna at 2 weeks of age just after ovulation and placed in ice-cold M4 medium containing $80 \mathrm{mM}$ sucrose (M4-sucrose). The synthesized dsRNA $\left(2 \mathrm{mg} \mathrm{ml}^{-1}\right)$ was mixed with $2 \mathrm{mM}$ Chromeo 494 fluorescent dye (Active Motif Chromeon $\mathrm{GmbH}$, Tegernheim, Germany), which was used as a visible marker for injection. Mixture containing $1 \mathrm{mg} \mathrm{ml}^{-1} \mathrm{dsRNA}$ was injected to eggs under a microscope. Then, injected eggs were transferred into new Petri dishes and incubated at $20^{\circ} \mathrm{C}$.

Construction of the reporter system. We used a two-hybrid system to detect heterodimerization of Met and SRC. Chinese hamster ovary cells were transfected according to the instructions ${ }^{32}$ with slight modifications. Dappu-Met(1-492) and Dapma-Met(1-497) were cloned into pBIND, and Dappu-SRC(1-600) and DappuSRC(1-666) (bHLH-PAS domain) were cloned into pACT (Promega). For transfections, Chinese hamster ovary cells were grown in 1:1 mixture of Dulbecco's Modified Eagle's Medium and Ham's F-12 containing 10\% fetal bovine serum, and incubated at $37^{\circ} \mathrm{C}$ in a $5 \% \mathrm{CO}_{2}$ atmosphere. The day before transfection, cells were transferred to a 24-well plate. The transfection was performed using FuGENE HD (Promega), according to the manufacturer's protocols. Each well received $0.05 \mathrm{mg}$ pBIND, $0.05 \mathrm{mg}$ pACT and $0.2 \mathrm{mg}$ pG5luc, which has luciferase gene under the control of GAL4-binding site. All juvenoids were dissolved in ethanol and added to the medium after $24 \mathrm{~h}$, and cells were harvested after $48 \mathrm{~h}$. Reporter activities were measured using the Dual-Luciferase Assay kit (Promega), according to the manufacturer's protocols. All transfections were performed at least three times, a

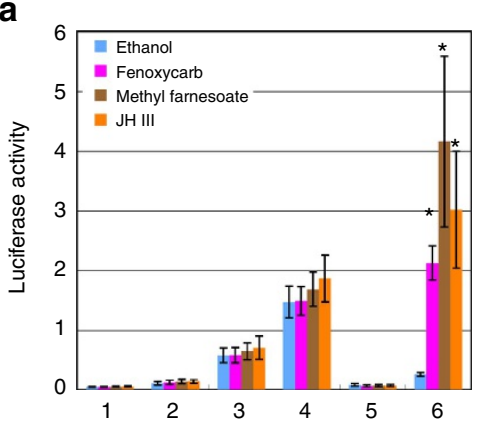

1. $\mathrm{pBIND}$

$x$ pACT

2. $p B I N D$

x pACT-Dappu-Met(1-492)

3. pBIND-Dappu-SRC(1-600)

$x$ pACT

4. $\mathrm{pBIND}-\mathrm{Dappu}-\mathrm{SRC}(1-600)$ x pACT-Dappu-SRC $(1-600)$

5. pBIND-Dappu-Met(1-492)

x pACT-Dappu-Met(1-492)

6. pBIND-Dappu-SRC(1-600)

x pACT-Dappu-Met(1-492)

\section{b}
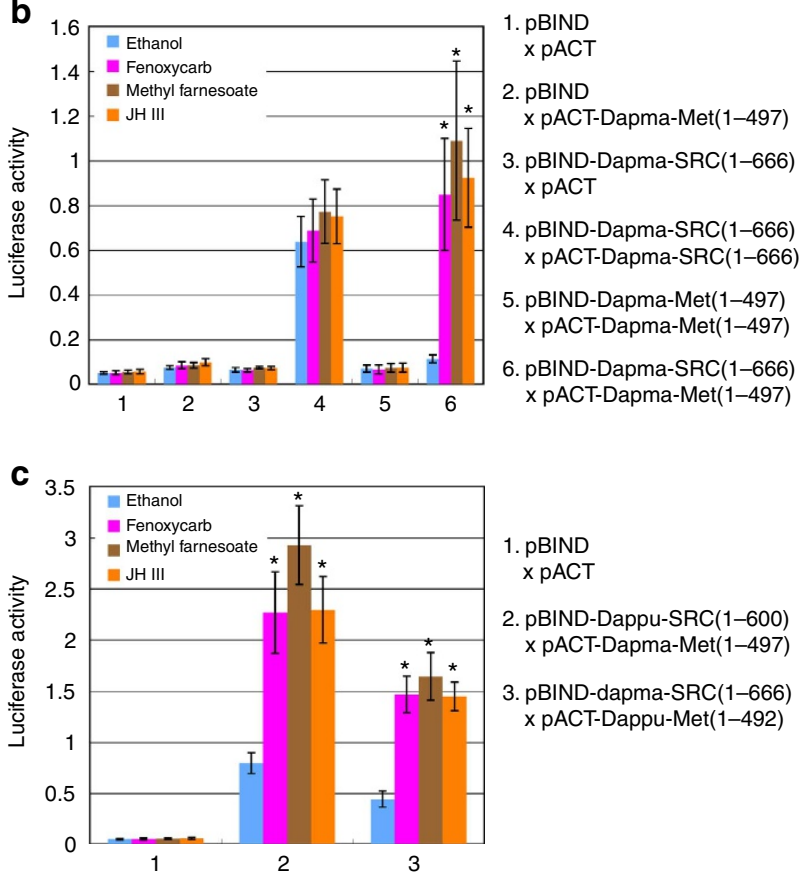

Figure 5 | Transcriptional activations with various vector combinations. (a) Response profile of $D$. pulex. (b) Response profile of $D$. magna. (c) Response profile for SRC and Met from different Daphnia species compared with each other. Vertical axis indicates luciferase activities. Each point represents the mean of triplicate determinations, and vertical bars represent the mean \pm s.d. Asterisks indicate significant differences $(P<0.05, t$-test $)$ from ethanol treatment of each vector combination. Concentrations of juvenoid $(\mathrm{M})$ used in this experiment are as follows: fenoxycarb (3.32E - 08), methyl farnesoate (3.99E-06) and JH III (3.75E - 05).

employing triplicate sample points in each experiment. The values shown are mean \pm s.d. from three separate experiments, and dose-response data and $\mathrm{EC}_{50}$ were analysed using GraphPad Prism (Graph Pad Software, Inc., San Diego, CA).

\section{References}

1. Nijhout, H. F. \& Wheeler, D. E. Juvenile hormone and the physiological basis of insect polymorphisms. Q. Rev. Biol. 57, 109-133 (1982).

2. Nijhout, H. F. Insect Hormones (Princeton University Press, 1994).

3. Nijhout, H. F. Control mechanisms of polyphenic development in insects. Bioscience 49, 181-192 (1999).

4. Hartfelder, K. \& Emlen, D. J. Comprehensive Molecular Insect Science Vol. 3 (eds Gilbert, L. I., Iatrou, K. \& Gill, S. S.) 651-703 (Elsevier, 2005).

5. Truman, J. W. et al. Juvenile hormone is required to couple imaginal disc formation with nutrition in insects. Science 312, 1385-1388 (2006).

6. Cornette, R., Gotoh, H., Koshikawa, S. \& Miura, T. Juvenile hormone titers and caste differentiation in the damp-wood termite Hodotermopsis sjostedti (Isoptera, Termopsidae). J. Insect. Physiol. 54, 922-930 (2008). 
7. Riddiford, L. M. Juvenile hormone action: a 2007 perspective. J. Insect. Physiol. 54, 895-901 (2008)

8. Olmstead, A. W. \& LeBlanc, G. A. Juvenoid hormone methyl farnesoate is a sex determinant in the crustacean Daphnia magna. J. Exp. Zool. 293, 736-739 (2002).

9. Olmstead, A. W. \& LeBlanc, G. A. Insecticidal juvenile hormone analogs stimulate the production of male offspring in the crustacean Daphnia magna. Environ. Health Perspect. 111, 919-924 (2003).

10. Tatarazako, N., Oda, S., Watanabe, H., Morita, M. \& Iguchi, T. Juvenile hormone agonists affect the occurrence of male Daphnia. Chemosphere $\mathbf{5 3}$ 827-833 (2003)

11. Arias, J. R. \& Mulla, M. S. Morphogenetic aberrations induced by a juvenile hormone analogue in the mosquito Culex tarsalis (Diptera: Culicidae). J. Med. Entomol. 12, 309-316 (1975).

12. Ali, A., Xue, R. D. \& Lobinske, R. Efficacy of two formulations of the insect growth regulator, pyriproxyfen (Nylar or Sumilarv), against nuisance Chironomidae (Diptera) in man-made ponds. J. Am. Mosq. Control Assoc. 9, 302-307 (1993)

13. Laufer, H., Ahl, J. S. B. \& Sagi, A. The role of juvenile hormones in crustacean reproduction. Am. Zool. 33, 365-374 (1993).

14. Helvig, C., Koener, J. F., Unnithan, G. C. \& Feyereisen, R. CYP15A1, the cytochrome P450 that catalyzes epoxidation of methyl farnesoate to juvenile hormone III in cockroach corpora allata. Proc. Natl Acad. Sci. USA 101, 4024-4029 (2004).

15. Shinoda, T. \& Itoyama, K. Juvenile hormone acid methyltransferase: a key regulatory enzyme for insect metamorphosis. Proc. Natl Acad. Sci. USA 100, 11986-11991 (2003).

16. Minakuchi, C., Namiki, T., Yoshiyama, M. \& Shinoda, T. RNAi-mediated knockdown of juvenile hormone acid O-methyltransferase gene causes precocious metamorphosis in the red flour beetle Tribolium castaneum. FEBS J. 275, 2919-2931 (2008).

17. Niwa, R. et al. Juvenile hormone acid O-methyltransferase in Drosophila melanogaster. Insect. Biochem. Mol. Biol. 38, 714-720 (2008).

18. Rider, C. V., Gorr, T. A., Olmstead, A. W., Wasilak, B. A. \& LeBlanc, G. A. Stress signaling: coregulation of hemoglobin and male sex determination through a terpenoid signaling pathway in a crustacean. J. Exp. Biol. 208, 15-23 (2005).

19. Eads, B. D., Andrews, J. \& Colbourne, J. K. Ecological genomics in Daphnia: stress responses and environmental sex determination. Heredity 100, 184-190 (2008).

20. Miyakawa, H. et al. Gene up-regulation in response to predator kairomones in the water flea, Daphnia pulex. BMC Dev. Biol. 10, 45 (2010).

21. Oda, S., Kato, Y., Watanabe, H., Tatarazako, N. \& Iguchi, T. Morphological changes in Daphnia galeata induced by a crustacean terpenoid hormone and its analog. Environ. Toxicol. Chem. 30, 232-238 (2011).

22. Oda, S., Tatarazako, N., Watanabe, H., Morita, M. \& Iguchi, T. Production of male neonates in Daphnia magna (Cladocera, Crustacea) exposed to juvenile hormones and their analogs. Chemosphere 61, 1168-1174 (2005).

23. Kleiven, O. T., Larsson, P. \& Hobaek, A. Sexual reproduction in Daphnia magna requires three stimuli. Oikos 65, 197-206 (1992).

24. Charles, J. P. et al. Ligand-binding properties of a juvenile hormone receptor, Methoprene-tolerant. Proc. Natl Acad. Sci. USA 108, 21128-21133 (2011).

25. Li, M., Mead, E. A. \& Zhu, J. S. Heterodimer of two bHLH-PAS proteins mediates juvenile hormone-induced gene expression. Proc. Natl Acad. Sci. USA 108, 638-643 (2011).

26. Zhang, Z. L., Xu, J. J., Sheng, Z. T., Sui, Y. P. \& Palli, S. R. Steroid receptor coactivator is required for juvenile hormone signal transduction through a bHLHPAS transcription factor, methoprene tolerant. J. Biol. Chem. 286, 8437-8447 (2011).

27. Miura, K., Oda, M., Makita, S. \& Chinzei, Y. Characterization of the Drosophila Methoprene-tolerant gene product. Juvenile hormone binding and liganddependent gene regulation. FEBS J. 272, 1169-1178 (2005).
28. Konopova, B. \& Jindra, M. Juvenile hormone resistance gene Methoprenetolerant controls entry into metamorphosis in the beetle Tribolium castaneum. Proc. Natl Acad. Sci. USA 104, 10488-10493 (2007).

29. Abdou, M. A. et al. Drosophila Met and Gce are partially redundant in transducing juvenile hormone action. Insect Biochem. Mol. Biol. 41, 938-945 (2011).

30. Godlewski, J., Wang, S. L. \& Wilson, T. G. Interaction of bHLH-PAS proteins involved in juvenile hormone reception in Drosophila. Biochem. Biophys. Res. Commun. 342, 1305-1311 (2006).

31. Regier, J. C. et al. Arthropod relationships revealed by phylogenomic analysis of nuclear protein-coding sequences. Nature 463, 1079-1083 (2010).

32. Kato, Y. et al. Cloning and characterization of the ecdysone receptor and ultraspiracle protein from the water flea Daphnia magna. J. Endocrinol. 193, 183-194 (2007).

33. Yin, C. M. et al. Identification of juvenile hormone III bisepoxide $\left(\mathrm{JHB}_{3}\right)$, juvenile hormone III and methyl farnesoate secreted by the corpus allatum of Phormia regina (Meigen), in vitro and function of $\mathrm{JHB}_{3}$ either applied alone or as a part of a juvenoid blend. J. Insect Physiol. 41, 473-479 (1995).

34. Darrouzet, E., Rossignol, F. \& Couillaud, F. The release of isoprenoids by locust corpora allata in vitro. J. Insect Physiol. 44, 103-111 (1998).

35. Betts, M. J. \& Russell, R. B. Bioinformatics for Geneticists (eds Barnes, M. R. \& Gray, I. C.) 289-316 (Wiley, 2003).

36. Thompson, J. D., Higgins, D. G. \& Gibson, T. J. CLUSTAL W: improving the sensitivity of progressive multiple sequence alignment through sequence weighting, position-specific gap penalties and weight matrix choice. Nucleic Acids Res. 22, 4673-4680 (1994).

37. Tamura, K. et al. MEGA5: molecular evolutionary genetics analysis using maximum likelihood, evolutionary distance, and maximum parsimony methods. Mol. Biol. Evol. 28, 2731-2739 (2011).

38. Kato, Y. et al. Development of an RNA interference method in the cladoceran crustacean Daphnia magna. Dev. Genes Evol. 220, 337-345 (2011).

39. Leo, C. \& Chen, J. D. The SRC family of nuclear receptor coactivators. Gene 245, 1-11 (2000).

\section{Acknowledgements}

The D. magna and D. pulex sequence data were provided by The Center for Genomics and Bioinformatics at Indiana University, which is supported in part by the METACyt Initiative of Indiana University, funded in part through a major grant from the Lilly Endowment, Inc. and distributed via wFleaBase in collaboration with the Daphnia Genomics Consortium (http://daphnia.cgb.indiana.edu). This study was partly supported through a grant from the Ministry of Education, Culture, Sports, Science and Technology (T.I.), the Ministry of the Environment of Japan (T.I.), a grant of Long-Range Research Initiative by Japan Chemical Industry Association (T.I.) and a grant from National Institute for Basic Biology (T.I.). H.M. and K.T. were supported by the Japan Society for the Promotion of Science (JSPS) research fellowship for young scientists. This study benefits from and contributes to the Daphnia Genomics Consortium.

\section{Author contributions}

H.M., S.M., Y.O. and T.I. designed the experiments. H.M. and K.T. performed the experiments. H.M., K.T., I.H. and S.O. analysed the data. S.O., N.T., T.M. and J.K.C discussed/commented on results and edited the manuscript. H.M., J.K.C. and T.I. wrote the paper. All authors have read and approved the final manuscript.

\section{Additional information}

Competing financial interests: The authors declare no competing financial interests.

Reprints and permission information is available online at http://npg.nature.com/ reprintsandpermissions/

How to cite this article: Miyakawa, H. et al. A mutation in the receptor Methoprene-tolerant alters juvenile hormone response in insects and crustaceans. Nat. Commun. 4:1856 doi: 10.1038/ncomms2868 (2013). 